\title{
Research on Space Positioning Model Based on Sun Shadow Trajectory
}

\author{
Yinping Zhou ${ }^{1}$ \\ 1 North China Electric Power University, School of Electrical and Electronic Engineering, Beijing,
}

102206

346591653@163.com

KEYWORDS: Sun Elevation Angle; Sun Shadow Positioning; Control Variable; Curve Fitting; Correlation Coefficient

\begin{abstract}
Research on how to use the video or image sequence variation in the shadow of the sun to determine the time and space that captures the time and place locate problems, combined with solar elevation angle formula, established a shadow the length $\mathrm{L}$ and the latitude $\varphi$, longitude $\lambda$, sun angle $\delta$, height $H$ and other objects of the mathematical relationship model.
\end{abstract}

\section{Introduction}

For a long shadow modeling, use of solar elevation angle, the sun was high and the inclination long shadow geometry, the first control variable method were studied long shadow with latitude, longitude, date changing with these three variables, and analyzes the reasons for this change, summarizes the changes in the characteristics. After combining the data subject, the model has been established, with the matlab draw a straight bar the shadow of the sun over the length of time curve.

Shadow vertex coordinates for spatial orientation and circumstances known at the time of the shooting problem, the model has three unknowns: $H, \varphi, \lambda$, you first need to add constraints curve fitting to solve, and then use the residuals squared correlation coefficient $\mathrm{R}$ is expressed or intended combined precision, a mathematical model (mathematical model and shadow change the latitude and longitude) straight bar location is given for solving analysis method steps and use the software 1stOpt with Matlab simulation analysis. The results showed that the use of Annex 1 to the data, obtained the corresponding shooting position Latitude: $19.2767^{\circ}$, Longitude: $108.6584^{\circ}$. (Hainan)

Temporal and Spatial Orientation for shadow vertex coordinate data and stem length under the circumstances known to the rod length model, the global software 1stOpt general method of Marquardt method, add multiple curve fitting optimization constraints to give each $\delta$ value corresponds to two dates. And calculate the correlation coefficient close to 1, the results show that the model is applied to solve a straight rod location and date is feasible.

For lower rod length with sun and shadow change dynamic information known case, identify problems video shooting location. First with matlab function in Video Reader reading out video information, and then extract the vertex coordinates of the pixel data using bar cursor in matlab calculate shadow length. If the date is known, a similar problem two methods give a prediction 
place; if the date is unknown, since the image measurement error exists, when global optimization is not convergence, PSO fitting two prediction place and date. ,

Finally, this paper summarizes the research on temporal and spatial positioning of the sun's shadow trajectory analysis, this paper sun shadow positioning technology principle, the mathematical model of the shadow length changes, the analysis with regard to the length of the shadow of the object height, variation of latitude and longitude, date, time. Case analysis shows temporal localization model based on the sun's shadow trajectory analysis is a better feasibility.

\section{Reiteration}

Determine the location and date of the video is one of the important applications of video data analysis, the sun shadow positioning technology is based on the length of the shadow of the sun changes the object in the video to determine video shooting location and date. The use of technology to solve the following problems:

1. The issue of a mathematical model of the shadow length changes, and calculate the Beijing Tiananmen Square (latitude 39 degrees 54 minutes 26 seconds east longitude 116 degrees 23 minutes 29 seconds) on a $3 \mathrm{~m}$ straight bar the shadow of the sun changes with time curve.

2. According to Annex 2 in the data1.mat and mathematical models to determine all the possible positions of a straight bar.

3. According to Annex 3 in the data2.mat and data3.mat data, model building, to determine if a straight bar all possible positions and date.

4. The combination of video, video capture model sites, determine all possible location, date unknown in further determine the shooting date and location.

\section{Problem Analysis}

\section{A. Analysis about Problem 1}

Problem 1 requires a change in the length of the shadow of a mathematical model to analyze the shadow length variation of each parameter. First, access to literature, to give the length of the shadow of the two main variables related to an elevation angle of the sun, one object height, there is a relationship among the equation. Then to draw the model according to October 22, 2015 Beijing time 9: 00-15: 00 Tiananmen Square (latitude 39 degrees 54 minutes 26 seconds east longitude 116 degrees 23 minutes 29 seconds) 3m high straight bar Sun curve shadow length. According to the definition of solar elevation angle, the use of these data and software Matlab, the curve can be obtained.

\section{B. Analysis about Problem 2}

Problem 2 requires sun shadow vertex coordinate data according to a fixed straight rod, a mathematical model to determine the location where the straight bar. And the model is applied to the data in Annex 2 data1.mat, given all the possible locations. Two basic problems of the mathematical model is still the sun elevation angle, high shadow, was the relationship between high. As the solar elevation angle trigonometric formulas are, there may be a plurality of solutions, so straight bar may have more than one location. Using software 1stOpt with Matlab, by curve fitting optimization, it can be determined straight bar possible locations.

\section{Analysis about Problem 3}

Problem 3 requirements according to a fixed straight bar vertex coordinates in a horizontal plane 
of the shadow of the sun, a mathematical model to determine the straight lever which place and date, combined with Annex 3 data2.mat and data3.mat data to determine the straight lever all possible date and venue. Question three is to further deepen the two issues, because the date in question two measurement data are known. Mathematical model of three basic problems still have to seize this fundamental solar elevation angle, in the curve fitting by increasing the number of constraints, using the global common law Marquardt algorithm calculates a straight bar possible date and venue.

\section{Analysis about Problem 4}

Problem 4 requirements of Annex combine video 2m Straight determine which locations. Four different issues and problems between two known data is not available, the need to obtain indirectly through the video. First, the use of image processing techniques to convert video from one frame to the picture, according to the scaling relations, we can obtain the coordinates of the vertices straight bar shadow, thereby establishing a mathematical model of the video shoot location, use 1stOpt with Matlab determine all possible video filming locations. There are deeper questions IV taken place at the same time and date unknown, at this time is more complicated mathematical model, combined with a variety of software, you can get a video of the shooting date and location.

\section{Model}

1stOpt software uses Marquardt method and the general global optimization algorithm optimization algorithm, the most important feature of these algorithms is to overcome the algorithm in the field of optimization calculation using an iterative method must be given appropriate initial value problem too subjective, we do not give the initial parameters value, given by the random 1stOp software, through its unique global optimization algorithm, eventually finding the optimal solution, while the software is embedded in a variety of other intelligent algorithms such as particle swarm optimization, annealing algorithm, genetic algorithm. Therefore, we use the fitting 1stOpt, data processing and drawing terms using matlab.Over the years, China's college English teaching has always.

A stationary rod straight day in the shadow length is constantly changing, due to the rotation of the earth and the earth revolves around the sun causes. 24 hours a day, a week of Earth's rotation $360^{\circ}$, the position of the sun is also changing over time, causing the changing straight bar shadow. According to the principle of relative motion, etc., can be determined straight bar like place and time information, which is the sun shadow positioning technology. The sun rises in the east, over time, continue to move south, eventually landing from the west, at noon shadow to a minimum.

According to the theoretical definition, the solar elevation angle $\alpha$, and the sun was high $\mathrm{H}$ relationship shadow length $\mathrm{L}$ is shown as below. Trigonometric, can be obtained:

$$
H=L \times \tan (\alpha)
$$

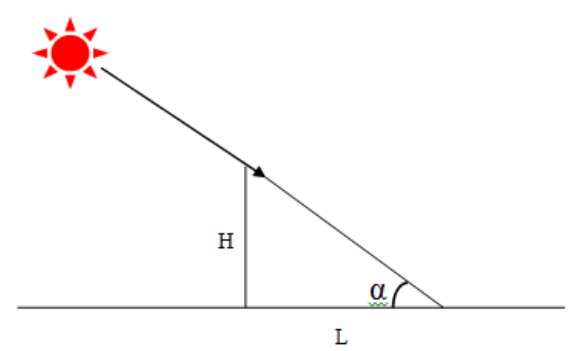

Fig 1. Forming a schematic shadow 
The relationship between shadow and shadow length the coordinates of the endpoints: (in straight bar at the bottom of the origin of coordinates).

One problem is given:

Date October 22, 2015, corresponding to the model in terms of latitude 39 degrees 54 minutes 26 seconds, corresponding to the model in terms of longitude 116 degrees 23 minutes 29 seconds, converted to the corresponding model, Beijing time 9: 00-15: 00 That is, the object height meters.

Combined with the above data and model, October 22, 2015 Beijing time 9: 00-15: 00

Tiananmen Square (latitude 39 degrees 54 minutes 26 seconds east longitude 116 degrees 23 minutes 29 seconds) sun shadow 3m high straight pole the length of the curve using Matlab, the results shown in Figure 2.

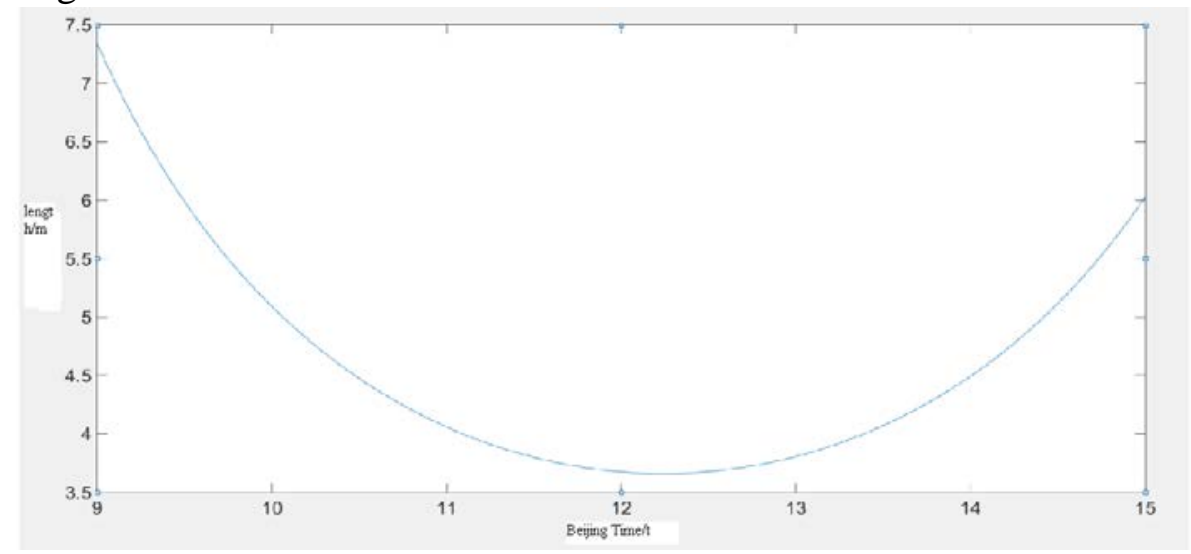

Fig 2. The change versus of straight sun shadow length with time

\section{Conclusion}

The mathematical model of the shadow length changes, the analysis with regard to the length of the shadow of the object height, variation of latitude and longitude, date, time, and the model established plotted October 22, 2015 Beijing time 9: 00: 00-15 Tiananmen Square (latitude 39 degrees 54 minutes 26 seconds east longitude 116 degrees 23 minutes 29 seconds) curve sun shadow length 3-meter straight rod.

According to the mathematical model (1) established, combined with data attachments, global optimization model curve fitting to obtain unknown parameters in the model, namely the longitude, latitude, in order to determine the position of a straight rod.

According to the mathematical model front, with (2), based on the date and the latitude and longitude are unknown, global optimization and a number of constraints to obtain all the unknown parameters to determine the straight lever all the possible location and date.

First two minutes to extract every video picture by picture sequence and lever length determines the length of the shadow.

Still using a mathematical model above, since the image measurement error exists, when global optimization does not converge, so the switch to fit particle swarm algorithm, solve all unknown parameters, determine the straight lever all the possible location and date.

\section{REFERENCE:}

[1]Schaaf, C.B., Wang, Z., Strahler, A.H. Commentary on Wang and Zender-MODIS snow albedo bias at high solar zenith angles relative to theory and to in situ observations in Greenland [J]. Remote Sensing of Environment: An Interdisciplinary Journal, 2011, 115(5) 
[2]Hoshang Kolivand, Mohd Shahrizal Bin Sunar.A New Application for Real-Time Shadow and Sun's Position in Virtual Environment [M]. Springer Berlin Heidelberg.2011.

[3] Ph. BlancL.Wald, L.Wald.The SG2 algorithm for a fast and accurate computation of the position of the Sun for multi-decadal time period [J]. Solar Energy.2012,86 (10)

[4] Richard Kittler. Stanislav Darula. Determination of time and sun position system [J]. Solar Energy. 2013, 93(Jul.)

[5]Schowengerdt, R. A. (2007). "Optical radiation models". Remote Sensing. pp. 45-88. Doi :10.1016/B978-012369407-2/50005-X. ISBN 9780123694072.

[6]U.S. Naval Observatory; U.K. Hydrographic Office, H.M. Nautical Almanac Office (2008). The Astronomical Almanac for the Year 2010. U.S. Govt. Printing Office. p.C5. ISBN978-0-7077-4082-9.

[7]Sproul, Alistair B. "Derivation of the solar geometric relationships using vector analysis. Retrieved 28 February 2012.

[8] J. W. Spencer (1971). "Fourier series representation of the position of the sun.

[9] Meeus, Jean (1991). "Chapter 12, Transformation of Coordinates". Astronomical Algorithms. Willmann Bell, Inc., Richmond, VA. ISBN0-943396-35-2. 